\title{
Steroidogenic Factor 1 (NR5A1) resides in centrosomes and maintains genomic stability by controlling centrosome homeostasis
}

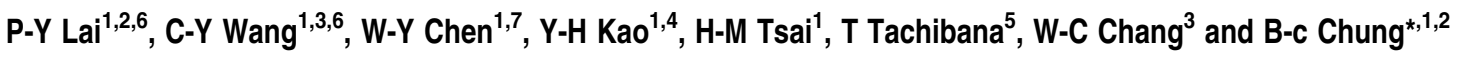

SF-1 (Steroidogenic Factor 1, NR5A1) is a tissue-specific transcription factor critical for the growth, development and differentiation of steroidogenic and a few other endocrine tissues. But how SF-1 regulates cell growth is not entirely clear. Here we found that SF-1 was localized to the centrosome in addition to the nucleus, and SF-1 depletion by shRNA caused centrosome over-duplication, aberrant mitosis and genomic instability, leading to a reduction of cell number. Centrosome amplification defect was rescued by both wild-type SF-1 and transcription-defective SF-1-G35E, suggesting a non-genomic activity of SF-1 involved in centrosome homeostasis. In addition, we identified in SF-1 a centrosome localization signal, whose overexpression led to reduced localization of both SF-1 and $\gamma$-tubulin to the centrosome. Our results uncover a novel role of SF-1 in the control of centrosome homeostasis and genomic stability.

Cell Death and Differentiation (2011) 18, 1836-1844; doi:10.1038/cdd.2011.54; published online 13 May 2011

SF-1 (Steroidogenic Factor 1, Ad4BP or NR5A1) is a member of the nuclear receptor family composed of a DNA-binding domain, a hinge region and a ligand-binding domain. ${ }^{1}$ As a transcription factor, SF-1 binds to specific DNA sequences and activates the expression of its target genes. ${ }^{2}$ These target genes are involved in steroidogenesis, reproduction, differentiation and sex determination in the adrenals, gonads and the brain. ${ }^{1,3}$

SF-1 regulates the development and differentiation of endocrine and reproductive organs. ${ }^{4}$ Sf-1 null-mice die shortly after birth; their adrenal glands and gonads are absent due to the apoptosis of adrenogenital primordial cells during embryonic development. ${ }^{5}$ Sf-1 heterozygous mice are impaired in adrenal growth following unilateral adrenalectomy. ${ }^{6}$ Clinically, SF-1 mutations causing reduced transcriptional activity are associated with the disorder of sex development and sometimes adrenal insufficiency. ${ }^{7}$ These studies indicate the requirement of adequate SF-1 activity for proper growth and differentiation of adrenals and gonads.

Contrary to SF-1 insufficiency, increased SF-1 dosage triggers adrenocortical cell proliferation. $S f-1$ transgenic mice develop adrenal tumors, and increased SF-1 dosage causes hyper-proliferation of adrenocortical H295 cells. ${ }^{8}$ Childhood adrenocortical tumors are also associated with increased copy number of SF-1 gene. ${ }^{9}$ The growth-promoting property of SF-1 can be reversed by treating cells with SF-1 inverse agonists. ${ }^{10}$ These studies demonstrate the importance of
SF-1 dosage and activity in adrenal growth. Data from patients, mouse models and cell lines all suggest the requirement of SF-1 activity for its function in growth and differentiation, although the detailed mechanism by which $\mathrm{SF}-1$ controls these processes is still unclear.

Cell growth is a tightly controlled process regulated by many factors. One such machinery is the centrosome. Centrosome contains a pair of centrioles and the surrounding pericentriolar materials (PCM). Centrioles are composed of nine microtubule triplets with $\alpha$ - and $\beta$-tubulin heterodimer as the basic building block. The PCM contains many proteins including $\gamma$-tubulin ring complex required for microtubules nucleation. In interphase, centrosomes are the primary microtubule organization center. During mitosis, centrosomes form asters that nucleate mitotic spindles for equal separation of chromosomes. ${ }^{11}$ Besides, the centrosome can serve as a scaffold to anchor a variety of regulatory proteins, which also contribute to cell cycle progression. ${ }^{12}$ Proper centrosome functions ensure faithful transmission of genetic information.

Centrosome homeostasis is required to maintain genetic fidelity during cell division. ${ }^{13}$ Similar to chromosomes, centrosomes duplicate only once in each cell cycle. ${ }^{14}$ If this duplication control is disrupted, centrosomes will be amplified. ${ }^{15}$ Amplified centrosomes will cause multipolar mitosis and chromosome mis-segregation, resulting in genomic instability. ${ }^{13,16}$ The maintenance of centrosome homeostasis is an important issue for proper cell growth.

\footnotetext{
${ }^{1}$ Institute of Molecular Biology, Academia Sinica, 115 Taipei, Taiwan; ${ }^{2}$ Institute of Molecular and Cellular Biology, Department of Life Science, National Tsing-Hua University, 300 Hsinchu, Taiwan; ${ }^{3}$ Institute of Biochemical Sciences, College of Life Science, National Taiwan University, 106 Taipei, Taiwan; ${ }^{4}$ Department of Biomedical Science and Technology, College of Life Science, National Taiwan University, 106, Taipei, Taiwan and ${ }^{5}$ Department of Bioengineering, Graduate School of Engineering, Osaka City University, Osaka, Japan

${ }^{*}$ Corresponding author: B-c Chung, Institute of Molecular Biology, Academia Sinica, 128 Academia Road Section 2, Nankang, Taipei 115, Taiwan.

Tel: + 886227899215 ; Fax: + 8862 27826085; E-mail: mbchung@ sinica.edu.tw

${ }^{6}$ These authors contributed equally to this work.

${ }^{7}$ Current address: Laboratory of Biochemistry and Molecular Biology, The Rockefeller University, New York, NY 10065, USA

Keywords: adrenal growth; centrosome amplification; NR5A1; $\gamma$-tubulin; centrosome localization signal

Abbreviations: BrdU, bromodeoxyuridine; SF-1, Steroidogenic Factor 1; PCM, pericentriolar material; CLS, centrosome localization signal; HU, hydroxyurea; $\gamma$-tub, $\gamma$-tubulin

Received 10.1.11; revised 04.4.11; accepted 06.4.11; Edited by B Zhivotovsky; published online 13.5.11
} 
a

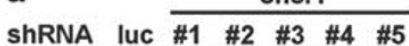

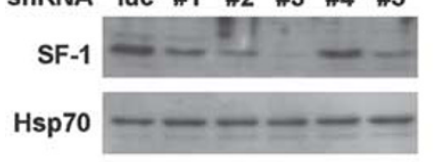

b

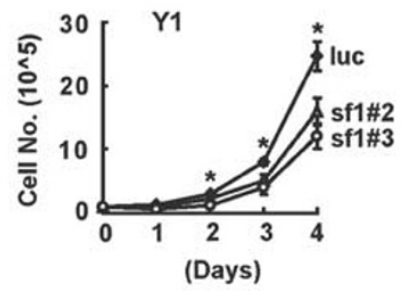

C

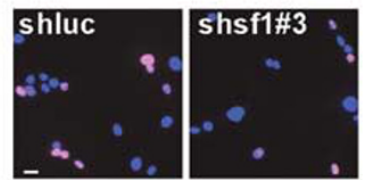

d

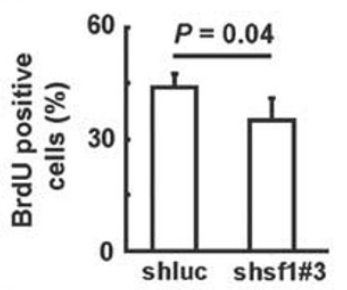

e

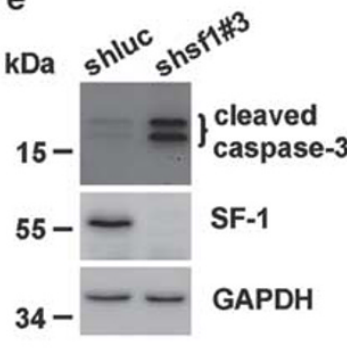

Figure 1 SF-1 depletion in Y1 cells caused cell growth delay, reduced cell proliferation and increased apoptosis. (a) Analysis of shRNA efficiency against SF-1 by immunoblotting. Proteins from cells infected with lentivirus encoding shRNA against luciferase (luc, as a control shRNA) or SF-1 (shsf1 \#1 to \#5) were analyzed for expression using antibodies against SF-1. Hsp70 was used as an internal control. (b) Growth curve of $Y 1$ cells after infection with shRNA-encoding lentivirus. ${ }^{*} P<0.05$ comparing with control (luc) group. (c) Examination of cell proliferation by $\mathrm{BrdU}$ incorporation. Merged pictures of cells stained with BrdU antibodies (pink) and co-stained with DAPI (blue) are shown. (d) Quantitation of BrdU positive cells. More than 200 cells from (c) were counted each time from four independent experiments and the mean \pm S.D. is shown. (e) Detection of apoptosis by immunoblotting with antibodies against cleaved caspase 3

In the present report, we have studied the role of SF-1 in growth control and found SF-1 resided in the centrosome in addition to the nucleus. A centrosome localization signal (CLS) at a.a. 348-367 of SF-1 was identified. Overexpression of this CLS polypeptide prevented the accumulation of SF-1 and $\gamma$-tubulin in the centrosome. We also found SF-1 depletion from adrenocortical $\mathrm{Y} 1$ cells caused centrosome over-duplication and subsequent genomic instability. Thus, we illustrated a novel function of SF-1 in maintaining centrosome homeostasis.

\section{Result}

SF-1 depletion leads to reduced cell growth and aberrant mitosis. To study the function of SF-1 in cell growth, we used shRNA delivered into mouse adrenocortical $Y 1$ cells by lentivirus to deplete SF-1 expression. A total of five different SF-1 shRNA sequences were tested. SF-1 shRNA \#2 and \#3 were most efficient in depleting SF-1 (Figure 1a). The effects of SF-1 depletion on $\mathrm{Y} 1$ cell growth were further examined. The number of $\mathrm{Y} 1$ cells depleted of SF-1 by shsf1 \#2 and shsf1 \#3 were significantly lower than those by control shluc (Figure 1b). These results indicated that SF-1 participated in $\mathrm{Y} 1$ cell growth control.

To understand how SF-1 depletion caused reduction of cell numbers, we examined cell proliferation by measuring bromodeoxyuridine (BrdU) incorporation and apoptosis by detecting cleaved caspase 3. Five days after lentivirus infection, cell proliferation as measured by BrdU incorporation was decreased (Figures 1c and d). Besides, apoptosis was increased as revealed by the higher levels of cleaved caspase 3 (Figure 1e). These results indicate that SF-1 depletion causes impaired cell growth by reducing cell proliferation and increasing apoptosis.

We further examined mitotic $Y 1$ cells by immunofluorescence at five days after lentivirus infection (Figure 2A). During mitosis, chromosomes from control luc cells lined up along the metaphase plate and bipolar spindle poles were detected by $\gamma$-tubulin staining (Figure 2Ba). But higher proportions of SF-1 depleted sf1 \#2 and sf1 \#3 cells contained disorganized chromosome that did not align on the metaphase plate with pseudo-bipolar (Figure 2Bb) or multi-polar (Figure 2Bc) spindle poles (Figure 2C). Enlarged nuclei (Figure 2D) were also detected in a greater fraction of SF-1-depleted cells (Figure 2E), and these cells with enlarged nuclei expressed a high level of senescence-associated $\beta$-galactosidase (Figure 2F). These results demonstrate that long-term SF-1 depletion leads to mitotic problems like formation of aberrant spindle poles, enlarged nuclei and eventually senescence, implying a role of SF-1 in maintaining genomic stability.

We examined SF-1-depleted cells in interphase using $\gamma$-tubulin as a centrosome marker. Many $\gamma$-tubulin spots were observed after SF-1 depletion (Figure 2G). The numbers of cells with multiple centrosomes were increased after SF-1 was depleted by shsf1 \#2 and shsf1 \#3 (Figure $2 \mathrm{H}$ ), indicating that SF-1 depletion leads to formation of multiple centrosomes.

SF-1 depletion causes centrosome over-duplication. SF-1 depletion led to the formation of both mitotic defects and multiple centrosomes. To differentiate the primary cause of these defects, we examined cells at an earlier time point (two days after lentivirus infection) (Figure 3a). Centrosome amplification was evident (Figure $3 b$ ), but these cells did not have enlarged nuclear size (Figure $3 c$ ) or multiple nuclei (Figure $3 d$ ). These results imply that the immediate defect caused by SF-1 depletion is centrosome amplification, and mitotic defects should be the consequence caused by centrosome amplification.

Centrosome amplification can arise from centrosome fragmentation, cytokinetic failure or over-duplication. To examine whether the amplified centrosomes caused by SF-1 depletion were fragmented, the components of centriole and PCM were examined (Figure $3 e$ ). All $\gamma$-tubulin spots contained the centriolar marker centrin (upper panel) and $\alpha$-tubulin (middle panel, treated with nocodazole). The results indicate that these amplified centrosomes are intact containing both centrioles and PCM.

To test the microtubule nucleating function of the centrosome, nocodazole was applied to depolymerize all microtubules. After removal of nocodazole allowing microtubule repolymerization, $\alpha$-tubulin threads were detected extending from the base of $\gamma$-tubulin spots, indicating that all these amplified centrosomes are capable of nucleating microtubule networks in vivo (lower panel). Thus, these amplified centrosomes retained the function to nucleate microtubules in interphase. 
A

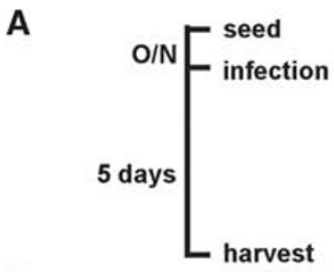

B

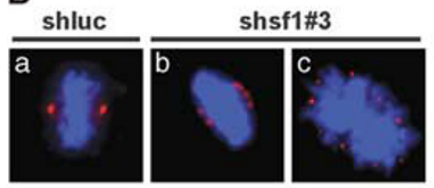

C
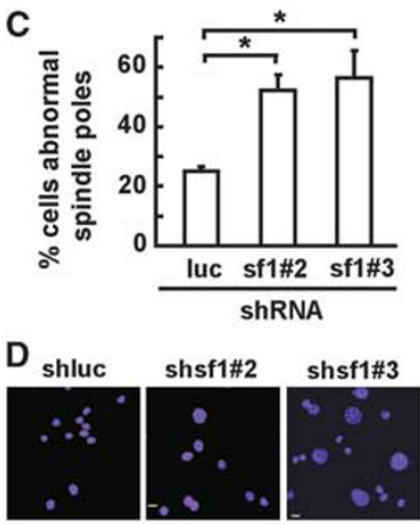

E

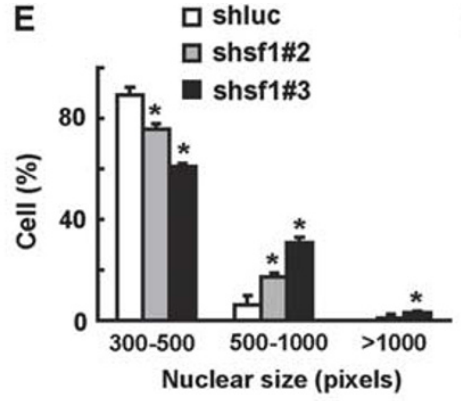

G

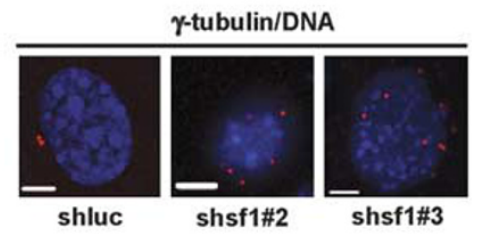

H

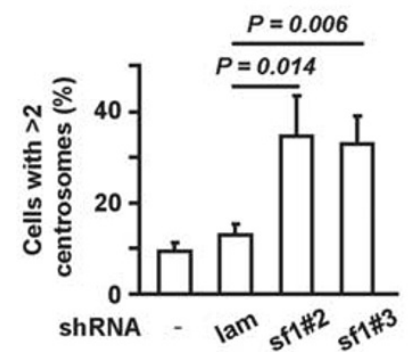

$\mathbf{F}$
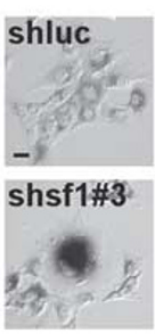

men

sf1\#3

Figure 2 Long-term SF-1 depletion in Y1 cells leads to aberrant mitosis and centrosome amplification. (A) Schematic representation of the experimental procedure used, except for $(\mathbf{F})$, which had a longer duration. After seeding and overnight $(\mathrm{O} / \mathrm{N})$ incubation, cells were infected with lentivirus encoding control (lamin, lam or luc) or SF-1 (\#2 and \#3) shRNA and incubated for five more days before harvesting. (B) Immunofluorescence examination of mitotic cells by staining with DNA (DAPI, blue) and spindle poles ( $\gamma$-tubulin, red). (C) Quantitation of mitotic cells with aberrant spindle poles. More than 50 mitotic cells in (D) were counted in three independent experiments. ${ }^{*} P<0.05$ comparing with control group. (D) Examination of nuclear size by DAPI staining. The scale bar is $5 \mu \mathrm{m}$. (E) Quantitation of nuclear areas. The areas of nuclei from at least 100 cells in (D) were counted and compared in three independent experiments. ${ }^{*} P<0.05$ comparing the value with control (luc) group within the same nuclear area. $(\mathbf{F})$ Cell senescence analysis after SF-1 depletion for 9 days. Scale bar is $20 \mu \mathrm{m}$. (G) Immunofluorescence detection of centrosomes by $\gamma$-tubulin antibody (red) and DNA by DAPI (blue). Scale bars are $5 \mu \mathrm{m}$. (H) Quantitative results of percentage of cells with aberrant centrosome numbers ( $>2$ ). At least 100 cells were counted in three independent experiments and the mean \pm S.D. is shown

To further examine the cause of centrosome amplification, we stained centrosomes with CEP170, which is present only in the mother centriole. ${ }^{17}$ Only one CEP170 signal was detected in each control shluc cell. SF-1 depletion by shsf1 \#2 or shsf1 \#3 did not change the number of CEP170 signals per cell, despite the presence of multiple $\gamma$-tubulin spots (Figures $3 f$ and $g$ ). The presence of a single mother centriole among many amplified centrosomes indicates that centrosome amplification caused by SF-1 deficiency is not due to cytokinetic failure, and is possibly due to centrosome over-duplication.

Centrosomes are duplicated during $S$ phase under the control of CDK2 in coordination with DNA replication. ${ }^{18}$ To further confirm if centrosome over-duplication occurred in SF-1-depleted cells, Y1 cells were arrested in S phase by treatment with hydroxyurea $(\mathrm{HU})$ for $24 \mathrm{~h}$ to allow more time for centrosome reduplication. More shsf1 \#3 cells had amplified centrosomes than control shluc cells (Figure 3h). When treated with roscovitine, a CDK2 inhibitor, centrosome amplification induced by shsf1 \#3 was blocked even in the presence of $\mathrm{HU}$ (Figure $3 \mathrm{~h}$ ). These results show that centrosome amplification in SF-1 depleted $\mathrm{Y} 1$ cells is due to centrosome over-duplication, but not centrosome fragmentation or cytokinetic failure.
In addition to adrenocortical Y1 cells, one would wonder whether centrosomes in other types of cells were also controlled by SF-1. To test this, we transfected FLAG-SF-1 into osteosarcoma cell line U2OS (Supplementary Figure S1A). The population of FLAG-SF-1-transfected cells with multiple centrosomes was not different from that of EYFPtransfected control cells either before or after HU treatment (Supplementary Figure S1B). Therefore, SF-1 does not affect centrosome numbers in U2OS cells.

Non-genomic function of SF-1 in maintaining centrosome homeostasis. To further confirm the effect of SF-1 on centrosome homeostasis, we tested whether exogenous SF-1 can rescue the centrosome defect. Y1 cells were first infected with lentivirus-expressing GFP or FLAG-tagged shRNAresistant SF-1 (SF-1 ${ }^{R}$, Supplementary Figure S2A), cultured under neomycin selection until uninfected cells were dead, then infected again with shsf1 \#3 lentivirus for five days. In these cells the endogenous SF-1 was efficiently depleted by shRNA, in contrast the level of exogenous SF-1 ${ }^{\mathrm{R}}$ was not affected (Figure 4a). Cyp11a1, an SF-1 target, was also restored to a level similar to control shluc $\mathrm{Y} 1$ cells. 
a
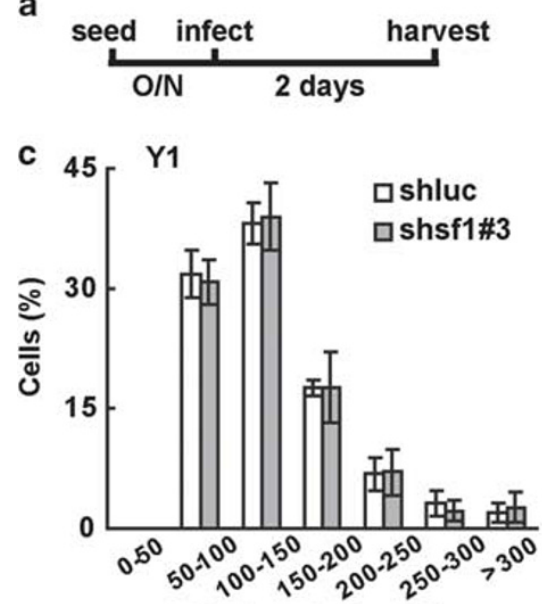

Nuclear size ( $\left.\mu \mathrm{m}^{\wedge} 2\right)$

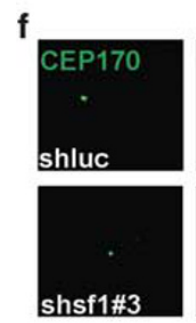

b

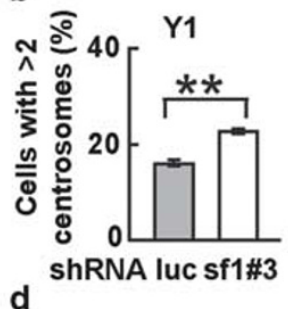

d

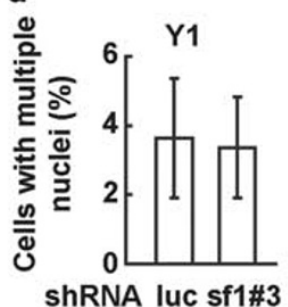

shRNA luc sf1\#3 e
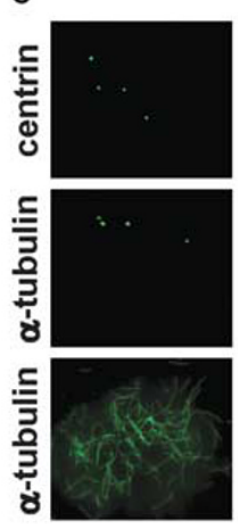

$\boldsymbol{\gamma}$-tubulin Merge/DNA
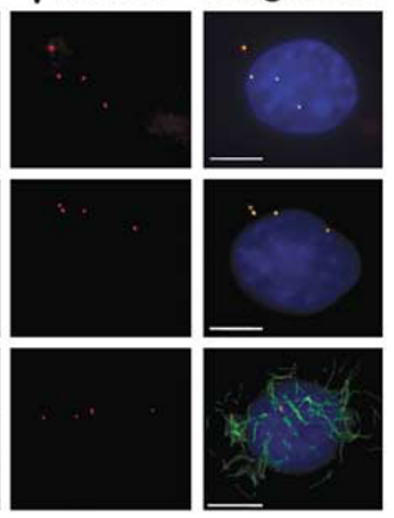

h

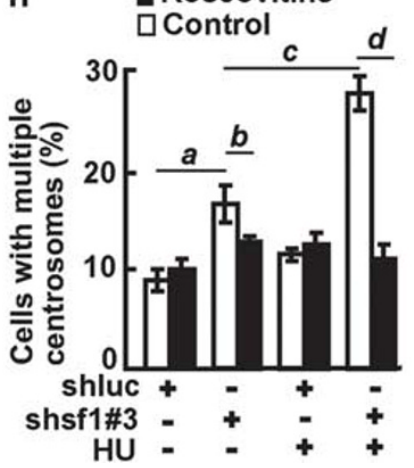

Figure 3 Centrosome amplification caused by short-term SF-1 depletion is due to centrosome over-duplication but not mitotic defects or centrosome fragmentation. (a) The diagram of experimental procedure. After seeding and overnight $(\mathrm{O} / \mathrm{N})$ incubation, cells were infected with lentivirus and incubated for two more days before harvesting. The numbers of centrosomes ( $\gamma$-tubulin staining) and areas of nuclei (DAPI staining) from at least 100 cells were counted in three independent experiments. (b) The percentages of cells with amplified centrosome $(>2) .{ }^{* *} P=0.0026$. (c) The distribution of nuclear areas of cells. (d) The percentages of cells with multiple nuclei ( $>$ 1). (e) Examination of centrosome integrity and function in SF-1-depleted Y1 cells. Immunostaining of centrosomes with $\gamma$-tubulin (middle column) and centrin (upper row) or $\alpha$-tubulin (middle and bottom rows) in shsf1 \#3 lentivirus-infected $\mathrm{Y} 1$ cells. Microtubules were depolymerized by nocodazole (middle and bottom rows) and followed by chase in fresh medium to repolymerize the microtubule networks (bottom row) before staining with $\alpha$-tubulin antibody. Scale bars are $5 \mu \mathrm{m}$. (f) Immunostaining of centrosomes with antibodies against CEP170 (green) and $\gamma$-tubulin (red) after Y1 cells were infected with control shluc (upper panel) or shsf1 \#3 (lower panel) lentivirus. Scale bars are $5 \mu$ m. (g) Cells containing one or more than one CEP170 spots were quantified after infection with the indicated lentivirus. (h) Cells containing multiple centrosomes were counted after the respective shRNA was delivered in the presence or absence of $\mathrm{HU}$ with (black bar) or without (white bar) roscovitine. $a, P=0.0000001 ; b, P=0.004 ; c, P=0.01$; $d, P=0.0002$. All results are expressed as the mean \pm S.D. from at least three independent experiments
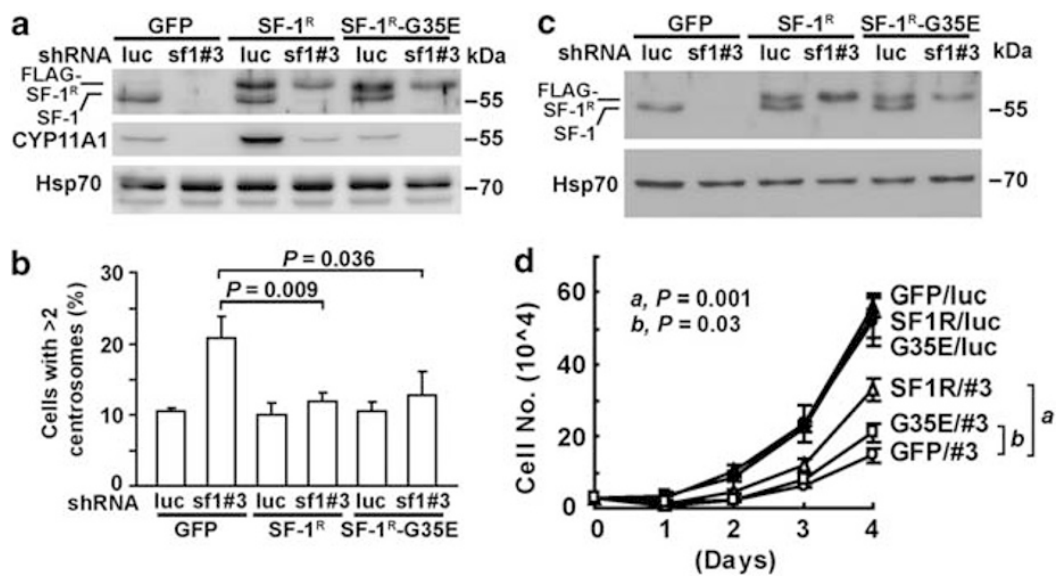

Figure 4 The G35E mutant of SF-1 can completely rescue the centrosome amplification defect and partially rescue the cell growth defect caused by SF-1 depletion. (a) Y1 derivatives expressing GFP, SF- $1^{\mathrm{R}}$ or SF-1 ${ }^{\mathrm{R}}$-G35E were infected with shluc or shsf1 \#3 lentivirus for five days. Levels of SF-1 and Cyp11a1 detected by immunoblotting using their respective antibodies are shown. Hsp70 immunoblotting is the loading control. (b) The percentage of cells from (a) with centrosome amplification ( $>2$ ) are shown. At least 100 cells were counted each time in three independent experiments and the mean \pm S.D. is shown. (c) Immunoblotting analysis of $Y 1$-derivatives stably expressing GFP, FLAG-SF-1 ${ }^{\mathrm{R}}$ WT or FLAG-SF-1 ${ }^{\text {R }}$-G35E infected with control (luc) or SF-1 (sf1 \#3) shRNA lentivirus. (d) Growth curve of Y1-derivatives infected with shRNA-encoding lentivirus. $a, P=0.001 ; b, P=0.03$ 
The ability of $\mathrm{SF}-1^{\mathrm{R}}$ to rescue centrosome amplification defect was examined. SF-1 depletion by shsf1 \#3 caused centrosome amplification in a high percentage of GFPexpressing $Y 1$ cells, but this number returned to the basal level when cells expressed SF-1 ${ }^{R}$ (Figure $4 b$ ). Besides this transient $\mathrm{SF}-1^{\mathrm{R}}$-expressing system, stable expression of $\mathrm{SF}-1^{\mathrm{R}}$ in $\mathrm{Y} 1$ also led to a reduction of the fraction of cells with multiple centrosomes even though its endogenous SF-1 was depleted by shsf1 \#3 (Supplementary Figure S2B). The rescue of the centrosome amplification defect by $\mathrm{SF}-1^{\mathrm{R}}$ demonstrates the function of SF-1 in preventing centrosome amplification.

To examine if the transcriptional activity of SF-1 is required to maintain centrosome homeostasis, we generated a lentivirus that expressed a mutant SF-1 without DNA-binding ability due to the replacement of its Gly35 by Glu (SF-1G35E). ${ }^{19}$ This G35E mutant was transcriptionally inactive, and when stably expressed in $\mathrm{Y} 1$ cells failed to induce Cyp11a1 expression (Figure 4a and Supplementary Figure $\mathrm{S} 2 \mathrm{C}$ ). It, however, rescued the centrosome defect induced by shsf1 \#3 (Figure 4b). This result indicates that the ability of SF-1 to prevent centrosome amplification is likely independent of its nuclear transcriptional activity.
We then determined if the cell growth delay caused by SF-1 depletion can be rescued by restoring SF-1 expression. $\mathrm{Y} 1$ cell clones stably expressing GFP, SF-1 ${ }^{R}$ and transcriptiondefective FLAG-SF-1 (SF-1 $\left.{ }^{\mathrm{R}}-\mathrm{G} 35 \mathrm{E}\right)$ with or without SF-1 depletion were tested (Figure $4 \mathrm{c}$ ). While control luc cells grew well, GFP-expressing cells depleted of SF-1 by sh \#3 grew poorly. Wild-type $S F-1^{R}$ significantly rescued the cell growth defect. The SF-1 ${ }^{\mathrm{R}}$-G35E also partially restored cell growth caused by SF-1 depletion (Figure 4d). This result suggests the involvement of transcription-independent function of SF-1 in maintaining cell growth in addition to the known transcriptiondependent function. ${ }^{8}$

Centriolar localization of SF-1. SF-1 is present in the nucleus. By immunofluorescence analysis we noticed a spot stained with SF-1 antibody adjacent to the nucleus of mouse adrenocortical $\mathrm{Y} 1$ cells (Figure $5 \mathrm{a}$ ). A monoclonal anti-SF-1 (7D5) antibody also detected the same staining pattern (Supplementary Figures S3A and B). This spot was colocalized with $\gamma$-tubulin, indicating it was a centrosome (Figure 5a and Supplementary Figure S3B). The same SF-1 staining was also observed in the centrosome of H295 cells a
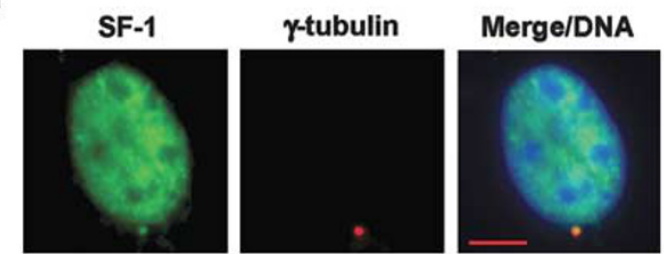

b

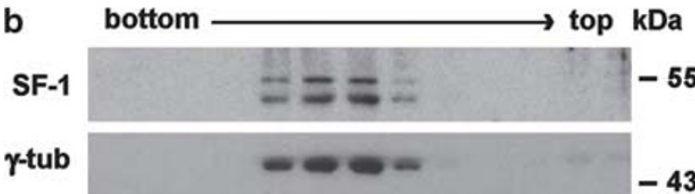

C
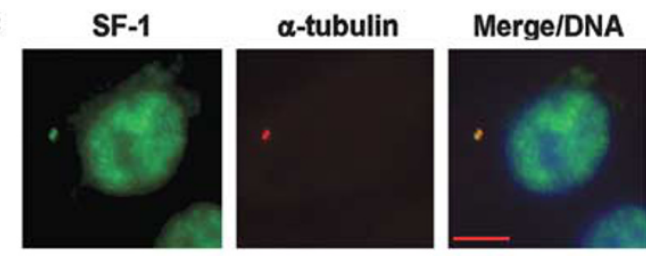

SF-1

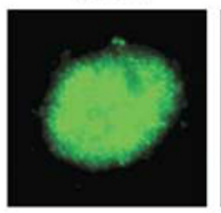

centrin

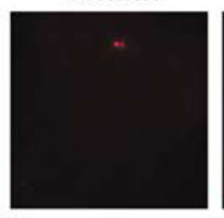

d
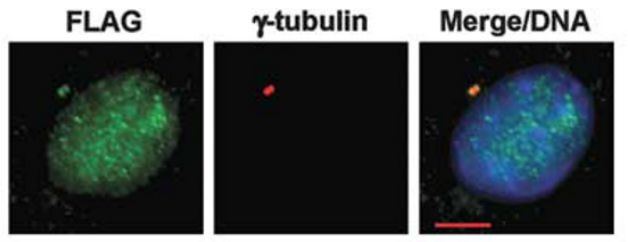

e

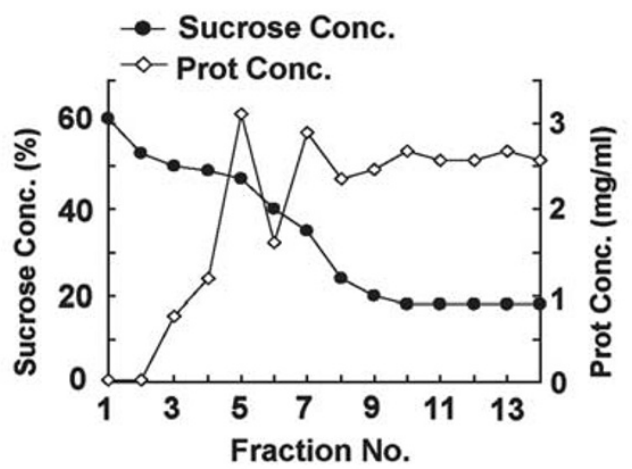

f

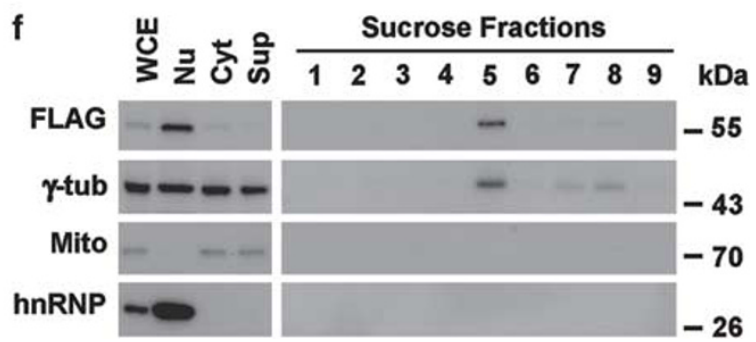

Figure 5 SF-1 is located in the centriole. (a) Immunofluorescence showing subcellular localization of SF-1 by staining with an antibody against SF-1 (green). Centrosomes are visualized by an antibody against $\gamma$-tubulin (red) in Y1 cells. The scale bar is $5 \mu \mathrm{m}$. (b) Sucrose gradient fractionation of centrosomal extract from Y1 cells. The fractionated centrosomal extract was analyzed by immunoblotting using antibodies against SF-1 or $\gamma$-tubulin $(\gamma$-tub). (c) Co-immunostaining with antibodies against SF-1 (green), $\alpha$-tubulin (red, upper panel, after treatment with nocodazole), or centrin (red, lower panel). Scale bars are $5 \mu \mathrm{m}$. (d) Immunofluorescence detection of FLAG-tagged SF-1 by anti-FLAG antibody and of centrosomes by anti- $\gamma$-tubulin antibody. The scale bar is $5 \mu \mathrm{m}$. (e) Sucrose gradient fractionation of centrosome extract from Y1 cells that have been transfected with FLAG-tagged SF-1. The curves represent the concentrations (Conc.) of sucrose (\%, solid circles) and protein (mg/ml, empty circles) of fractions. (f) Immunoblotting analysis of whole cell extract (WCE), nuclear fraction (Nu), cytoplasmic fraction (Cyt), and post-centrosomal supernatant (Sup) prepared from Y1 cells, as well as fractions from sucrose gradient fractionations. hnRNP, hnRNP A1; Mito, mitochondria complex II; $\gamma$-tub, $\gamma$-tubulin 
(Supplementary Figure S3C). The localization of SF-1 to the centrosome was further investigated by centrosome fractionation using sucrose gradient centrifugation (Figure 5b). SF-1 co-fractionated with $\gamma$-tubulin, further confirming the centrosome localization of SF-1 in $\mathrm{Y} 1$ cells.

The location of SF-1 within the centrosome was further examined by co-staining with the centriole markers $\alpha$-tubulin (Figure 5c, upper panel, with nocodazole treatment) and centrin (Figure 5c, lower panel). The colocalization of SF-1 with centriole markers indicates the presence of SF-1 in the centriole.

In addition to endogenous SF-1, transfected FLAG-tagged SF-1 was detected in the nucleus and centrosome using FLAG antibodies (Figure 5d). Sucrose gradient fractionation of the centrosome preparation showed that FLAG-SF-1 and $\gamma$-tubulin co-sedimented in the same fractions between $50 \%$ and $40 \%$ of sucrose (Figures $5 \mathrm{e}$ and $\mathrm{f}$ ). The absence of Mitochondrial Complex II and nuclear hnRNPA1 signals in the centrosome fractions excluded the possibility of nuclear and mitochondrial contaminations. Thus, our data show that SF-1 is localized to the centriole in addition to the nucleus.

\section{Identification of SF-1 centrosomal localization} signal. To identify the signal peptide that targets SF-1 to the centrosome, SF-1 was truncated into various fragments and fused with GFP for easy subcellular identification by green fluorescence. All those SF-1 fragments containing a.a. 348-367 were present in peri-nuclear dots (arrows in
Figure 6a) plus occasional nuclear or cytoplasmic distribution. Those peri-nuclear dots colocalized with $\gamma$-tubulin (Figure $6 \mathrm{~b}$ and Supplementary Figure S4A). Thus, we identified a CLS of SF-1 as a.a. 348-367. The ability of CLS to localize in the centrosome was further confirmed by fractionation of cell lysate and centrosome purification. GFPCLS, but not GFP, was present in the centrosome fraction together with $\gamma$-tubulin, confirming centrosome localization of this peptide (Figure 6c). Looking at this CLS polypeptide more closely, we found that it has repeated sequences of hydrophobic residues containing Leu or Val (Figure 6d). These amino acids were mutated to Ala, creating a $9 \mathrm{~A}$ mutant. GFP fused to the wild-type CLS, but not the 9A mutant, was localized to peri-nuclear centrosomal dots, indicating the importance of these amino acids in targeting SF-1 to the centrosome. We have mutated SF-1 at three, six, or nine residues of this CLS (Supplementary Figure S4B). All these mutants, however, lost transcriptional activities (Supplementary Figure S4B), thus precluding the separation of transcriptional activity from centrosome targeting.

To examine the function of this CLS, we overexpressed SF-1 CLS. Y1 cells expressing GFP-CLS had reduced SF-1 staining in the centrosome. When compared with untransfected cells or cells expressing GFP alone, these GFP-CLS cells also had reduced $\gamma$-tubulin signal in the centrosome (Figures $7 \mathrm{a}$ and $\mathrm{b}$ ). Microtubule regrowth after nocodazole treatment was also impaired after CLS overexpression a

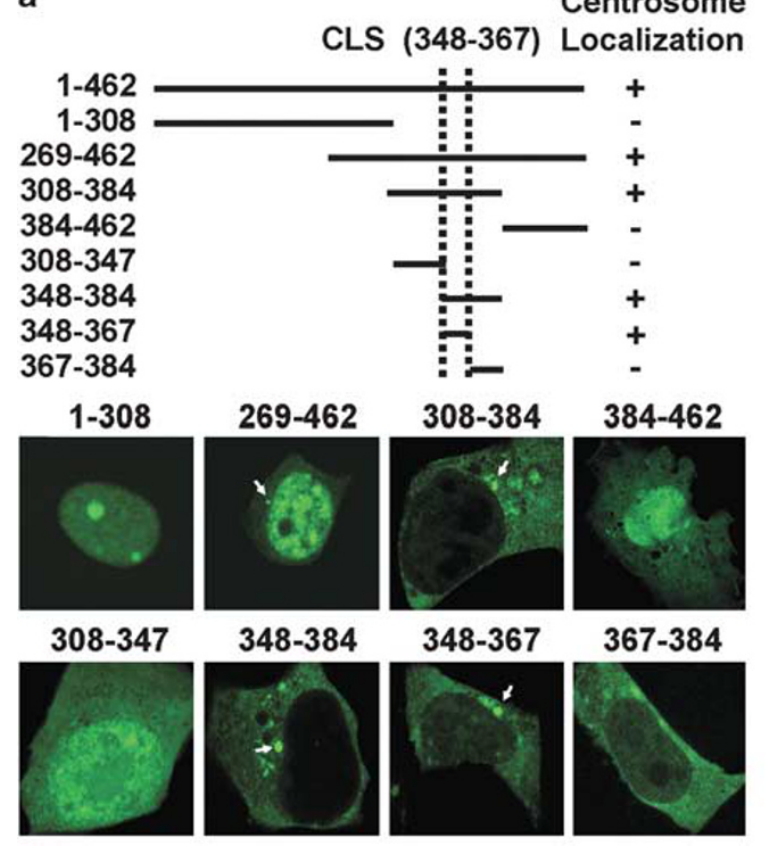

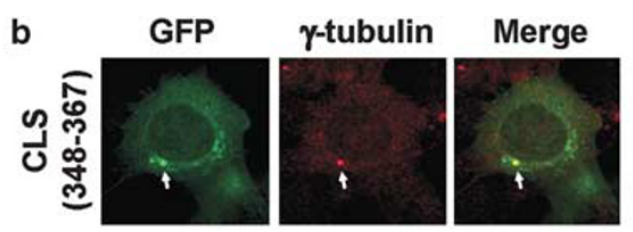

c

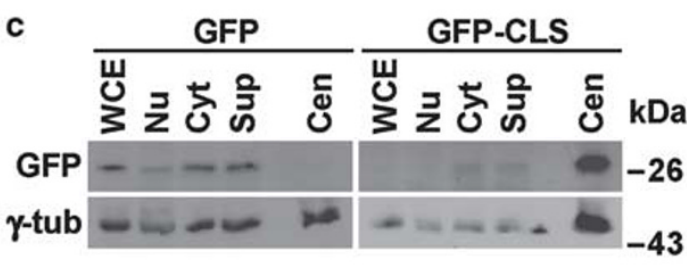

d

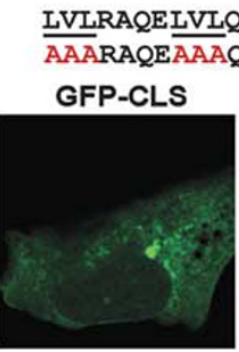
43

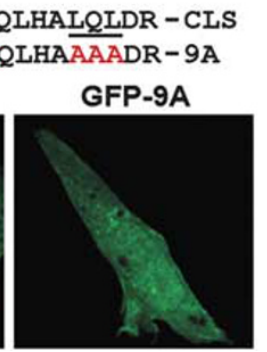

Figure 6 Identification of CLS in SF-1. (a) Schematic representation of various SF-1 fragments fused to GFP. Numbers denote the amino-acid residues in murine SF-1 (upper panel). Cells transfected with GFP-fused SF-1 fragments for $6 \mathrm{~h}$ were fixed and the fluorescence was examined by confocal microscopy. Arrows point to the centrosomes. Fragments showing presence or absence of centrosome localization are labeled with '+ ' or '-', respectively. The deduced CLS is located at a.a. 348-367. (b) Immunofluorescence analysis of GFP-CLS (a.a. 348-367). After transfection, cells were stained with $\gamma$-tubulin (red) and fluorescence was examined by confocal microscopy. Arrows indicate the location of centrosomes. (c) Immunoblot analysis of $Y 1$ cell fractions after transfection with GFP or GFP-CLS using antibodies against GFP or $\gamma$-tubulin ( $\gamma$-tub). WCE, whole cell extract; Nu, nuclear fraction; Cyt, cytoplasmic fraction; Sup, post-centrosomal supernatant; Cen, centrosome fraction. (d) Fluorescence detection of GFP-fused SF-1 CLS and its 9A mutant. The amino-acid sequences are listed 
a

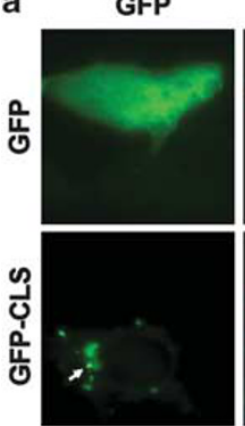

b

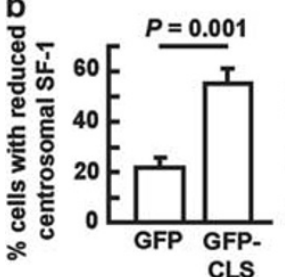

SF-1
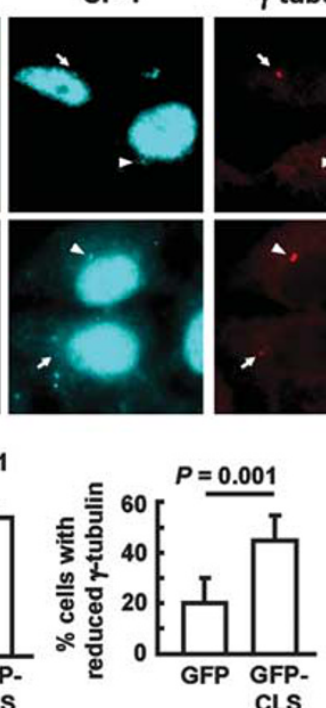

$\gamma$-tubulin

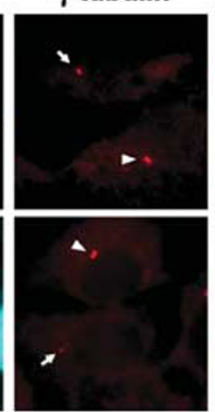

d
Merge/DNA
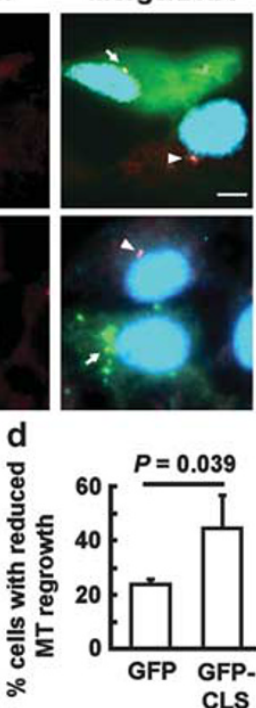

$P=0.039$

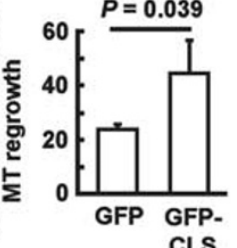

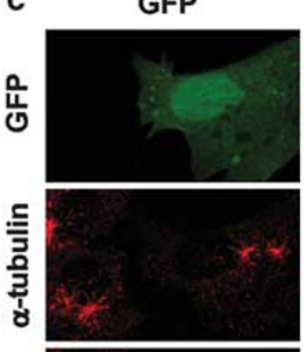

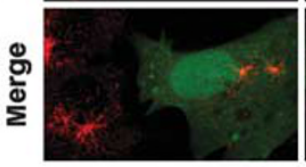

e

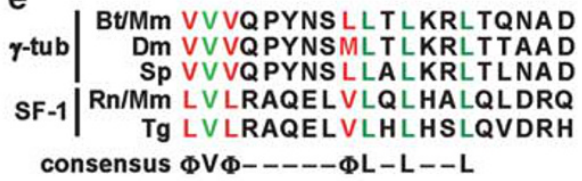

Figure 7 Reduced localization of SF-1 and $\gamma$-tubulin to the centrosome after SF-1 CLS overexpression. (a) Fluorescence detection of GFP or GFP-CLS, and co-stained with antibodies against SF-1 (cyan) and $\gamma$-tubulin (red). DNA (blue) was stained with DAPI. Scale bar is $5 \mu \mathrm{m}$. Arrows refer to the centrosomes of transfected cells, whereas arrowheads point to the centrosomes of untransfected cells. (b) Reduced centrosomal SF-1 (left panel) and $\gamma$-tubulin (right panel) signals in GFP-CLS-expressing cells. Around 100 cells were counted in three independent experiments. (c) Immunofluorescence detection of GFP or GFP-CLS (green). $\alpha$-Tubulin staining is in red. Scale bar is $5 \mu \mathrm{m}$. (d) Reduced microtubule (MT) regrowth in cells that express GFP-CLS. Around 60 cells were counted in three independent experiments and the mean \pm S.D. are shown. (e) Sequence alignment of SF-1 CLS (SF-1) and $\gamma$-tubulin ( $\gamma$-tub). Identical residues are shown in green, and similar ones in red. Species abbreviations: Bt, Bos taurus; $\mathrm{Mm}$, Mus musculus; Dm, Drosophila melanogaster, $\mathrm{Sp}$, Schizosaccharomyces pombe; Rn, Rattus norvegicus; Tg, Taeniopygia guttata. The putative consensus sequence is shown below. $\Phi$, hydrophobic amino acid

(Figures 7c and d), suggesting compromised centrosome function. The displacement of SF-1 and $\gamma$-tubulin from the centrosome by this CLS implies that these two proteins may share common features in centrosome localization. Indeed when sequences of SF-1 CLS and $\gamma$-tubulin from different species were aligned, a consensus motif was detected with conserved Val and Lue (Figure 7e), implying similar centrosome-targeting machineries that recognize this CLS. Thus, this CLS in SF-1 or a related region in $\gamma$-tubulin appears to target SF-1 and $\gamma$-tubulin to the centrosome for normal composition and function of centrosomes.

\section{Discussion}

In this study, we have investigated the function of SF-1 in maintaining centrosome homeostasis and genomic stability. We found that SF-1 was located in both nuclei and centrosomes, and SF-1 regulated centrosome homeostasis without invoking its transcriptional activity. We also identified a CLS sequence that targeted SF-1 to the centrosome. Overexpression of this CLS reduced accumulation of SF-1 and $\gamma$-tubulin in the centrosome. Our results reveal a new function of SF-1 in regulating centrosome homeostasis possibly through a non-genomic function.

Requirement of SF-1 in maintaining genomic stability and cell growth. In this report, we demonstrated the importance of SF-1 in maintaining adrenocortical $\mathrm{Y} 1$ cell growth. Our results are consistent with the reported role of SF-1 in adrenocortical cell proliferation. ${ }^{8}$ The function of SF-1 in centrosome regulation can at least partly explain the requirement of SF-1 in supporting steroidogenic cell growth by maintaining centrosome homeostasis and genomic stability. Furthermore, SF-1 overexpression in transgenic mice triggers cancer formation. ${ }^{8}$ Cancer has been tightly linked to centrosome abnormality, as amplified centrosomes cause mitotic defects like multipolar mitosis, chromosome mis-segregation or cytokinetic defects, contributing to genomic instability. ${ }^{13,16,20}$ Although the centrosome number in SF-1-overexpressing tumor has not been examined, it is conceivable that centrosome defects would exist as cancer cells are often associated with genomic instability caused by centrosomal defects.

The reduced cell number in vitro that we observed after SF-1 depletion correlates well with that in Sf-1-null mice. The adrenogenital primordial cells of $S f$-1-null mice undergo apoptosis at $\mathrm{E} 11.5-\mathrm{E} 12.5,{ }^{5}$ but the cause of this apoptotic death is still not understood. Our observation of apoptosis and centrosome amplification caused by SF-1 deficiency may explain why Sf-1-null adrenogenital primordial cells die, as centrosome amplification causes aberrant mitosis and subsequent cell cycle arrest or cell death. ${ }^{13,21}$ It would be interesting to examine whether the adrenogenital primordial cells of these Sf-1-null mice contain multiple centrosomes before they die. This experiment will not be easy because one has to catch the short time window at around E11.5 when these progenitor cells are dying.

SF-1 is a tissue-specific factor present mainly in the adrenals and gonads. Our results showed that SF-1 controlled centrosome numbers of only adrenocortical $\mathrm{Y} 1$ cells but not 
osteosarcoma U2OS cells. This result is consistent with the cell type specificity of SF-1. Growth properties from different cell types often vary. Each growing cell has its own cell typespecific factors that regulate cell cycle progression. For example, MyoD, a myoblast-specific factor, regulates the growth and differentiation of only muscle cells, ${ }^{22}$ while GATA1 regulates the growth and differentiation of erythroid cells. ${ }^{23}$ SF-1 is expressed mainly in the adrenal and gonad to regulate the growth of steroidogenic tissues. Our results illustrate a way by which a cell-specific protein controls the growth of a particular cell lineage. It would be of interest to examine the centrosomes from other cells and whether their growth is controlled by cell type-specific centrosomal proteins.

Role of SF-1 in centrosome homeostasis. SF-1 participates in the control of adrenogonadal cell growth. ${ }^{8}$ In the current studies, we found SF-1 also regulated centrosome homeostasis, and the G35E mutant of SF-1 retained the ability to control centrosome numbers. SF-1G35E is a mutant impaired in DNA binding and transcription activation. $^{24}$ Therefore, the transcriptional activity of SF-1 is likely not required for the control of centrosome homeostasis.

In addition to the transcriptionally impaired G35E mutation, we also examined CLS mutations of SF-1. Unfortunately all these CLS mutants lost their transcriptional activities. The CLS of SF-1 is located in a conserved region at the ligandbinding domain presumably important for transcription; this is probably why the abolishment of centrosome targeting also affects the transcriptional activity of SF-1. Despite this difficulty, centrosomal proteins often contribute to centrosome homeostasis. $^{12,25}$ The characterization of SF-1 as a centrosomal protein provides a possible mechanism for this nongenomic SF-1 function. Our results reveal a novel function of SF-1 in maintaining centrosome homeostasis possibly through its centrosomal, but not nuclear, localization.

In our results, SF-1 depletion-induced centrosome amplification is strongly enhanced in the presence of $\mathrm{HU}$ and can be reversed by treating with roscovitine, suggesting the uncoupling of centrosome duplication cycle and DNA duplication cycle upon SF-1 depletion. Recent report describes that centrosomes can still duplicate in the prolonged G1 phase induced by mimosine treatment without significant rise in CDK2 activity, providing another pathway for centrosome duplication. ${ }^{26}$ However, this situation probably does not occur in SF-1-depleted $Y 1$ cells because centrosome amplification caused by SF-1 depletion can be rescued by CDK2 inhibitor roscovitine.

Functional importance of SF-1 centrosome localization signal. In this report we identified a 20-amino-acid CLS sequence located at the ligand-binding domain that could target SF-1 to the centrosome. Overexpression of this CLS resulted in reduced accumulation of SF-1 and $\gamma$-tubulin in the centrosome. This CLS has similar properties as other centrosome-targeting domains from proteins like AKAP450, Cyclin A, Cyclin E, which displace their endogenous proteins from centrosomes when overexpressed. ${ }^{27-30}$ These CLS domains interact with scaffold proteins important for centrosome localization and functions of their endogenous proteins.
The CLS from SF-1 can displace both SF-1 and $\gamma$-tubulin from the centrosome, indicating that both proteins may share similar centrosome-targeting machineries. $\gamma$-Tubulin exists as complexes along with other proteins. ${ }^{31}$ Centrosomal localization of $\gamma$-tubulin complexes is mediated by multiple proteins such as pericentrin, AKAP450/CG-NAP, NEDD1/GCP-WD, ninein and ninein-like proteins through interaction with $\gamma$-tubulin complex components. ${ }^{31}$ The presence of shared amino acids in SF-1-CLS and $\gamma$-tubulin suggests the existence of common interacting proteins. SF-1-CLS overexpression may block the recruitment of $\gamma$-tubulin to centrosomes by competing for unidentified binding proteins. Searching for the SF-1-CLS interacting proteins can give us more hints on this phenomenon. The other possibility is that CLS in SF-1 might be shared by other proteins functioning in the centrosomal recruitment of $\gamma$-tubulin; thus, overexpression of SF-1-CLS may compete away those proteins involved in $\gamma$-tubulin recruitment. It would be interesting to identify those proteins that may contain similar functional domains like SF-1-CLS.

\section{Materials and Methods}

The experimental details for plasmids and their construction, cell culture, derivation of stable protein overexpression cells, cell growth assay, antibodies, NCBI accession number of the genes for sequence alignment, lentivirus-mediated RNA Interference and cDNA expression are provided in the Supplementary information.

Centrosome purification. Centrosome was purified from asynchronous $\mathrm{Y} 1$ cells by sucrose gradient ultra-centrifugation as described previously. ${ }^{32}$ Briefly, cells were treated with nocodazole $(10 \mu \mathrm{g} / \mathrm{ml})$ and cytochalasin B $(5 \mu \mathrm{g} / \mathrm{ml})$ for $60 \mathrm{~min}$ at $37^{\circ} \mathrm{C}$, then lysed in cold lysis buffer containing $10 \mathrm{~mm}$ Tris- $\mathrm{HCl}, \mathrm{pH} 8.0,0.5 \% \mathrm{NP}-40$ and $0.1 \% \beta$-mercaptoethanol. After centrifugation at $2000 \times g$, the supernatant was overlaid on a $50 \%$ sucrose $(\mathrm{w} / \mathrm{w})$ cushion layer and centrifuged again at $10000 \times g$ for $1 \mathrm{~h}$. Supernatant was removed and the concentrated centrosomes were separated by centrifugation over a discontinuous sucrose gradient containing 65,50 and $40 \%$ sucrose $(\mathrm{w} / \mathrm{w})$ at $35000 \times \mathrm{g}$. Samples $(400 \mu /$ fraction) were collected from the bottom of the tube.

Immuno-fluorescence microscopy. To visualize SF-1, $\gamma$-tubulin, centrin, and CEP170 by immunofluorescence, cells were harvested by direct fixation with ice-cold methanol for $5 \mathrm{~min}$ followed by antibodies staining. To visualize centrosomal FLAG-SF- 1 by anti-FLAG and depolymerized $\alpha$-tubulin by anti- $\alpha$ tubulin staining, cells were pretreated with $30 \mu \mathrm{m}$ nocodazole on ice for $1 \mathrm{~h}$ to depolymerize microtubule networks, followed by extraction with saponin $(20 \mathrm{ng} / \mathrm{ml})$ for $2 \mathrm{~min}$ before fixation. After fixation and blocking with $5 \%$ BSA, cells were incubated with antibodies overnight at $4^{\circ} \mathrm{C}$ followed by incubation with Alexa 488 , Alexa 546, or Alexa 633 conjugated secondary antibodies (Invitrogen, Carlsbad, CA, USA) for $1 \mathrm{~h}$ in the dark.

The nuclei were stained with 4, 6-diamino-2-phenylindole (DAPI, $0.1 \mu \mathrm{g} / \mathrm{ml}$ ). Fluorescence was detected with an Axiolmager Z1 fluorescence microscope or an LSM 510 confocal microscope (Zeiss, Zurich, Switzerland). The number of centrosomes and centrioles from more than one hundred cells was counted under the microscope. To measure nuclear size, DAPI signals were quantified using the MetaMorph software (Universal Imaging Corporation, West Chester, PA, USA). Student's $t$-test was used to analyze experiments within two groups as indicated. All data are expressed as the mean \pm S.D. from at least three independent experiments.

To identify SF-1 CLS, various SF-1 fragments fused to GFP were transfected into $\mathrm{Y} 1$ cells for $6 \mathrm{~h}$ followed by paraformaldehyde fixation, then analyzed by direct fluorescence examination of GFP and/or immunofluorescence detection of $\gamma$-tubulin.

In CLS overexpression experiment, cells were transfected with GFP or GFP-CLS plasmids for $18 \mathrm{~h}$ followed by fixation in ice-cold methanol and co-staining with SF-1 and $\gamma$-tubulin antibodies. Only GFP-positive cells without obvious aggregation were selected for quantitation to avoid the side effect caused by protein aggregation. The centrosomal SF-1 or $\gamma$-tubulin signals in the transfected cells were compared with 
those in the nearby non-transfected cells in the same field. The percentages of cells with significantly weaker signals were then scored.

Microtubule regrowth assay. Cells were washed with PBS once and incubated in drug-free medium at $37^{\circ} \mathrm{C}$ for 5 min after microtubule depolymerization by nocodazole $(10 \mu \mathrm{g} / \mathrm{ml})$ for $1 \mathrm{~h}$. After microtubule repolymerization at $37^{\circ} \mathrm{C}$ for $5 \mathrm{~min}$, cells were extracted with saponin and fixed with ice-cold methanol in SF-1 depletion experiments or directly fixed with paraformaldehyde without extraction in CLS experiments, then followed by immunofluorescence microscopy.

\section{Conflict of Interest}

The authors declare no conflict of interest.

Acknowledgements. We thank Jeffrey $L$ Salisbury and Chung Wang for the anti-centrin (2OH5) and Hsp70 antibodies, respectively. RNAi reagents were obtained from the National RNAi Core Facility (Institute of Molecular Biology/ Genomic Research Center, Academia Sinica, supported by National Research Program for Genomic Medicine, NSC 97-3112-B-001-016). This work was supported by grants from Academia Sinica and from National Science Council (NSC 97-2321-B-001-023), Taiwan.

1. Val P, Lefrancois-Martinez AM, Veyssiere G, Martinez A. SF-1 a key player in the development and differentiation of steroidogenic tissues. Nucl Recept 2003; 1: 8 .

2. Little TH, Zhang Y, Matulis CK, Weck J, Zhang Z, Ramachandran A et al. Sequencespecific deoxyribonucleic acid (DNA) recognition by steroidogenic factor 1 : a helix at the carboxy terminus of the DNA binding domain is necessary for complex stability. Mol Endocrinol 2006; 20: 831-843.

3. Parker KL, Rice DA, Lala DS, Ikeda Y, Luo X, Wong M et al. Steroidogenic factor 1: an essential mediator of endocrine development. Recent Prog Horm Res 2002; 57: 19-36.

4. Sadovsky $\mathrm{Y}$, Dorn C. Function of steroidogenic factor 1 during development and differentiation of the reproductive system. Rev Reprod 2000; 5: 136-142.

5. Luo X, Ikeda Y, Parker KL. A cell-specific nuclear receptor is essential for adrenal and gonadal development and sexual differentiation. Cell 1994; 77: 481-490.

6. Beuschlein F, Mutch C, Bavers DL, Ulrich-Lai YM, Engeland WC, Keegan $C$ et al. Steroidogenic factor-1 is essential for compensatory adrenal growth following unilateral adrenalectomy. Endocrinology 2002; 143: 3122-3135.

7. Lin L, Achermann JC. Steroidogenic factor-1 (SF-1, Ad4BP, NR5A1) and disorders of testis development. Sex Dev 2008; 2: 200-209.

8. Doghman M, Karpova T, Rodrigues GA, Arhatte M, De Moura J, Cavalli LR et al. Increased steroidogenic factor-1 dosage triggers adrenocortical cell proliferation and cancer. Mol Endocrinol 2007; 21: 2968-2987.

9. Figueiredo BC, Cavalli LR, Pianovski MA, Lalli E, Sandrini R, Ribeiro RC et al. Amplification of the steroidogenic factor 1 gene in childhood adrenocortical tumors. J Clin Endocrinol Metab 2005; 90: 615-619.

10. Doghman M, Cazareth J, Douguet D, Madoux F, Hodder P, Lalli E et al. Inhibition of adrenocortical carcinoma cell proliferation by steroidogenic factor-1 inverse agonists. J Clin Endocrinol Metab 2009; 94: 2178-2183.
11. Doxsey S. Re-evaluating centrosome function. Nat Rev Mol Cell Biol 2001; 2: 688-698.

12. Doxsey S, Zimmerman W, Mikule K. Centrosome control of the cell cycle. Trends Cell Biol 2005; 15: 303-311.

13. Fukasawa K. Oncogenes and tumour suppressors take on centrosomes. Nat Rev Cancer 2007; 7: 911-924.

14. Nigg EA. Centrosome duplication: of rules and licenses. Trends Cell Biol 2007; 17: 215-221.

15. D'Assoro AB, Lingle WL, Salisbury JL. Centrosome amplification and the development of cancer. Oncogene 2002; 21: 6146-6153.

16. Ganem NJ, Godinho SA, Pellman D. A mechanism linking extra centrosomes to chromosomal instability. Nature 2009; 460: 278-282.

17. Guarguaglini G, Duncan PI, Stierhof YD, Holmstrom T, Duensing S, Nigg EA et al. The forkhead-associated domain protein Cep170 interacts with Polo-like kinase 1 and serves as a marker for mature centrioles. Mol Biol Cell 2005; 16: 1095-1107.

18. Meraldi P, Lukas J, Fry AM, Bartek J, Nigg EA. Centrosome duplication in mammalian somatic cells requires E2F and Cdk2-cyclin A. Nat Cell Biol 1999; 1: 88-93.

19. Achermann JC, Ito M, Ito M, Hindmarsh PC, Jameson JL. A mutation in the gene encoding steroidogenic factor-1 causes XY sex reversal and adrenal failure in humans. Nat Genet 1999; 22: 125-126.

20. Nigg EA. Centrosome aberrations: cause or consequence of cancer progression? Nat Rev Cancer 2002; 2: 815-825.

21. Shang ZF, Huang $B, X u$ QZ, Zhang SM, Fan R, Liu XD et al. Inactivation of DNAdependent protein kinase leads to spindle disruption and mitotic catastrophe with attenuated checkpoint protein 2 phosphorylation in response to DNA damage. Cancer Res 2010; 70: 3657-3666.

22. Rudnicki MA, Schnegelsberg PN, Stead RH, Braun T, Arnold HH, Jaenisch R. MyoD or Myf-5 is required for the formation of skeletal muscle. Cell 1993; 75: 1351-1359.

23. Suwabe N, Takahashi S, Nakano T, Yamamoto M. GATA-1 regulates growth and differentiation of definitive erythroid lineage cells during in vitro ES cell differentiation. Blood 1998; 92: 4108-4118.

24. Ito M, Achermann JC, Jameson JL. A naturally occurring steroidogenic factor-1 mutation exhibits differential binding and activation of target genes. J Biol Chem 2000; 275: 31708-31714.

25. Cunha-Ferreira I, Bento I, Bettencourt-Dias M. From zero to many: control of centriole number in development and disease. Traffic 2009; 10: 482-498.

26. Durcan TM, Halpin ES, Casaletti L, Vaughan KT, Pierson MR, Woods S et al. Centrosome duplication proceeds during mimosine-induced G1 cell cycle arrest. J Cell Physiol 2008; 215: 182-191.

27. Keryer G, Witczak O, Delouvee A, Kemmner WA, Rouillard D, Tasken K et al. Dissociating the centrosomal matrix protein AKAP450 from centrioles impairs centriole duplication and cell cycle progression. Mol Biol Cell 2003; 14: 2436-2446.

28. Gillingham AK, Munro $S$. The PACT domain, a conserved centrosomal targeting motif in the coiled-coil proteins AKAP450 and pericentrin. EMBO Rep 2000; 1: 524-529.

29. Pascreau G, Eckerdt F, Churchill ME, Maller JL. Discovery of a distinct domain in cyclin A sufficient for centrosomal localization independently of Cdk binding. Proc Natl Acad Sci USA 2010; 107: 2932-2937.

30. Matsumoto Y, Maller JL. A centrosomal localization signal in cyclin E required for Cdk2independent S phase entry. Science 2004; 306: 885-888.

31. Raynaud-Messina B, Merdes A. Gamma-tubulin complexes and microtubule organization. Curr Opin Cell Biol 2007; 19: 24-30.

32. Bornens M, Paintrand M, Berges J, Marty MC, Karsenti E. Structural and chemical characterization of isolated centrosomes. Cell Motil Cytoskeleton 1987; 8: 238-249.

\section{Supplementary Information accompanies the paper on Cell Death and Differentiation website (http://www.nature.com/cdd)}

